\title{
Energetics in martensites
}

\section{Oscar P. Bruno}

Oscar P. Bruno, "Energetics in martensites," Proc. SPIE 3039, Smart Structures and Materials 1997: Mathematics and Control in Smart Structures, (13 June 1997); doi: 10.1117/12.276557 


\title{
Energetics in Martensites
}

\author{
Oscar P. Bruno \\ Applied Mathematics, 217-50, Caltech, Pasadena, CA 91125
}

\begin{abstract}
Martensitic transformations are shape-deforming phase transitions which can be induced in certain alloys as a result of changes in the imposed strains, stresses or temperatures. The interest in these alloys, which undergo a shape-deforming phase transition from a high temperature phase (austenite) to a low temperature phase (martensite), stems in part from their applicability as elements in active structures. In this paper we focus on the energy transfers that accompany the martensitic phase change. We discuss, in three concrete examples, the ways in which temperature, together with the elastic and dissipated energies, determine the equilibria as well as the quasi-static dynamics in martensites. Thus, in $\S 1$ we consider the pseudoelastic hysteresis in shape-memory wires; our treatment draws from [7,3]. In $\S 2$, on the other hand, we follow [4] and discuss equilibrium configurations in polycrystalline martensitic polycrystals. In §3, finally, we present some new theoretical computations for certain typical microstructural lengthscales, the twin widths, observed in single-crystalline martensite twinning.
\end{abstract}

\section{Shape-Memory Wires}

In this section we deal with the dynamics of shape-memory materials in one of its simplest settings, i.e., that of one dimensional wires under quasi-static deformations. The present discussion draws from recent experimental and theoretical work on NiTi wires [3,7], indicating that the pseudoelastic hysteresis in their strain-stress curves increases when the imposed strain rate is increased. Our model of these wires combines nonlinear thermoelasticity with a problem of heat flow determined by latent heat release at a two-phase interface. This model provides a law for the motion of a martensite-austenite interface which results, in particular, in excellent predictions of a variety of experimental results, as explained at the end of this section.

A number of models have been proposed for the study of the phenomenon of hysteresis in tension experiments involving SMA's. The experimental results mentioned above motivated us [7] to introduce yet another model of thermoelasticity. Our model, which is very simple indeed, can predict quantitatively experimental results such as those mentioned above. Points of contact as well as some essential differences between our approach and others are discussed in [2]. In this section a brief review of our approach is presented.

Our model is based on a set of four assumptions which have been discussed extensively, cf. [2, 3, 7]. These assumptions imply the following set of equations for the temperature profile $\theta(x, t)$ and the interface $s=s(t)$ in a wire of length $L_{0}$ :

$$
\begin{gathered}
\rho c_{p} \theta_{t}=k \theta_{x x}-\frac{2 h}{r}\left(\theta-\theta_{0}\right), \quad 0<x<\frac{L_{0}}{2}, \\
{\left[k \theta_{x}\right]_{-}^{+}(s(t), t)=-l^{*} \dot{s}(t)}
\end{gathered}
$$

and

$$
\left\{\begin{array}{lr}
\sigma=\left(\sigma_{M}(\theta(s(t), t)) \pm \sigma^{\text {hyst }}\right) & \text { for } \pm \dot{s}>0 \\
\left|\sigma-\sigma_{M}(\theta(s(t), t))\right| \leq \sigma^{\text {hyst }} & \text { for } \dot{s}=0
\end{array}\right.
$$

with initial and boundary conditions given by

$$
\theta(x, 0)=\theta_{0}, \quad s(0)=0 \text { and } \theta_{x}(0, t)=\theta_{x}\left(\frac{L_{0}}{2}, t\right)=0 .
$$


In equations (1) and (2), $\rho$ is the mass density of the unstrained wire, $c_{p}$ its specific heat, and $k, h$ and $r$ its thermal conductivity, coefficient of convection of heat and radius, respectively. Equations (1)-(4) reflect the experimental observation, mentioned above, of two interfaces, one starting from each end. Indeed, symmetry considerations on a wire of length $L_{0}$ show that we must have $\theta_{x}\left(\frac{L_{0}}{2}, t\right)=0$ at all times. The Maxwell stress $\sigma_{M}$ may safely be assumed to be a known linear function of temperature (which can be determined easily from very slow experiments), and the stress $\sigma$, finally, is determined by the overall elongation and the position $s(t)$ of the interface.
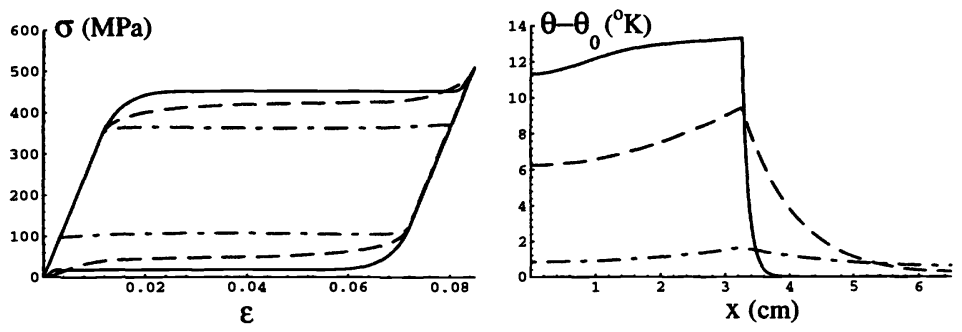

Figure 1: Theoretical predictions for a strain-controlled experiment of elongation of a NiTi wire of length $L_{0}=13 \mathrm{~cm}$ in air at three elongation rates $\dot{\epsilon} L_{0}$ : Solid curves, $50 \mathrm{~mm} / \mathrm{min}$; Dashed curves, $5 \mathrm{~mm} / \mathrm{min}$; Dot-dashed curves, $0.5 \mathrm{~mm} / \mathrm{min}$. Left.Strain stress curves. Right.- Temperature profiles corresponding to the half transformation time $2 \cdot s(t)=6.5 \mathrm{~cm}$.
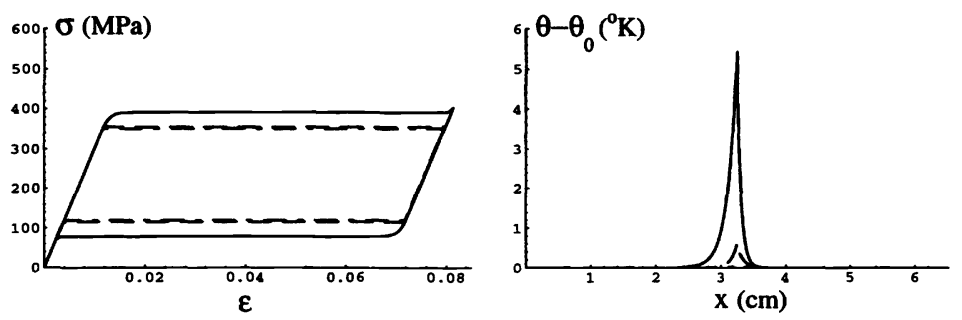

Figure 2: Same as in Figure 1, but for the experiments in water.

These equations may be used to model both strain controlled and stress controlled configurations. For sufficiently long wires it is possible to derive, from equations (1)-(4), an analytic expression relating the hysteresis width as a function of the imposed strain or stress rates:

$$
l^{*} v=\delta \theta \sqrt{\left(\rho c_{p} v\right)^{2}+8 h k / r}
$$

see [3]. Table 1 gives a comparison of theoretical and experimental results. The second and third columns in this table correspond to numerical simulations of wires of length $L$ with $L=13 \mathrm{~cm}$ and $L=78 \mathrm{~cm}$. Inspection of these figures shows that the $L=13 \mathrm{~cm}$ wire used in the experiments is already in the asymptotic domain of validity of equation (5). The experimental results are those of [7]; the agreement between theory and experiment falls within the experimental error. 


\begin{tabular}{ccccc}
\hline$L_{0} \dot{\epsilon}$ & $L=13 \mathrm{~cm}$ & $L=78 \mathrm{~cm}$ & Eqn. (5) & Expt. \\
\hline 0.5 in water & 350.4 & 350.4 & 350.4 & $345-355$ \\
5 in water & 354.5 & 354.5 & 354.5 & $350-360$ \\
50 in water & 391.2 & 391.3 & 391.2 & $370-400$ \\
0.5 in air & $362-364$ & 361.1 & 361.1 & $355-375$ \\
5 in air & $415-425$ & 425.1 & 425.0 & $420-440$ \\
50 in air & 451.1 & 451.3 & 450.9 & $450-470$ \\
\hline
\end{tabular}

Table 1: Strain-controlled experiments. Comparison between the asymptotic stresses $\sigma$ (in $\mathrm{MPa}$ ) obtained from numerical solutions, from equation (5) and from experiment for various imposed elongation rates $L_{0} \dot{\epsilon}($ in $\mathrm{mm} / \mathrm{min}$ ).

In Figures 1 and 2 we show strain-stress curves and the temperature profiles predicted by our theory. These curves were obtained by numerical integration of equations (1)-(4) by the method of lines [8]; comparison with the experimental curves of [7] again shows good agreement. The temperature profiles shown on the right side of the Figures are snapshots taken at the time for which half of the wire had transformed into martensite.

Further confirmation of our theory has been given by the experiments of Shaw and Kyriakides [9] and Shield, Leo and Grebner [10]. The first of these papers shows a variety of experiments for wires in water and in air, with temperature measurements in close agreement with those given above. The experiments of [10], finally, test the validity of the theory in a stress controlled configuration. For this case our theory predicts the existence of a critical stress above which the speed of transformation is infinite. This prediction was confirmed and, further, very good agreement of predicted and observed stresses and strain rates was found.

\section{Polycrystals and Martensitic Transformations}

The patterns of transformation in martensitic polycrystals result as a compromise between two factors. On one hand they recognize a tendency which, in order to avoid conflicts with the imposed boundary conditions, would have the grains transform with an average transformation strain as close as possible to the applied strain. A second tendency, on the other hand, would have the grains not transform at all in order to avoid increases in energy resulting from mismatch between the transformation strains of neighboring grains. In $\S 2.2$ we discuss numerical calculations and rigorous bounds for overall elastic energies in martensitic polycrystals. Such overall energies should prove useful in a finite element studies of macroscopic phenomena, such as the occurrence of two (macroscopic) interfaces, mentioned in the previous section, which occur during the elongation of polycrystalline NiTi wires.

\subsection{Elastic Energy}

We consider a polycrystal consisting of disjoint crystallites $G^{(1)}, G^{(2)} \ldots$, covering all space, each one of which can undergo a shape-deforming phase transition. Each grain in this martensitic polycrystal can undergo a phase deforming phase transition and it transforms, under given solicitations, in order to reduce the total elastic energy in the structure. To characterize the possible modes of transformation of the various grains it suffices to prescribe the set $\mathcal{S}$ of all strains that may arise as a result of transformation of a fixed single crystal whose axes are, say, parallel to the coordinate axes. The set of admissible transformation strains in a given grain $G^{(n)}$ is given, then, by conjugation of the set $\mathcal{S}$ by the rotation matrix associated with its crystallographic orientation and restriction to $G^{(n)}$. In a two dimensional case, for example, calling

$$
Q(\theta)=\left[\begin{array}{cc}
\cos (\theta) & \sin (\theta) \\
-\sin (\theta) & \cos (\theta)
\end{array}\right]
$$

the planar rotation of angle $\theta$, the set of admissible transformation strains in the grain $G^{(n)}$ is given by

$$
\mathcal{S}^{(n)}=\left(Q^{t}\left(\theta^{(n)}\right) \mathcal{S} Q\left(\theta^{(n)}\right)\right)
$$


see [4] for a more detailed mathematical description of random polycrystals.

In this paper we make the simplest assumption that all the phases are isotropic with identical elastic constants; these will be denoted by

$$
\nu=\text { Poisson Ratio and } \mu=\text { Shear Modulus. }
$$

Assume a homogeneous displacement

$$
\epsilon_{i j}^{0} x_{j}
$$

is applied on the boundary of our body $B$. Further, let $\epsilon^{T}(x, \omega)$ be a given admissible assignment of transformation strains, and call $u=u(x)=u(x, \delta, \omega)$ the displacement which results from both the boundary displacement and the set of transformation strains $\epsilon^{T}(x, \delta)=\epsilon^{T}\left(\frac{x}{\delta}\right)$ on the rescaled grains $G^{(n), \delta}=\delta G^{(n)}$. In the homogenization limit $\lim _{\delta \rightarrow 0}$ the elastic energy produced by a given distribution $\epsilon^{T}$ of transformation strains under given boundary conditions is given by

$$
W=W_{\epsilon^{T}}=\lim _{\delta \rightarrow 0} W_{\epsilon^{T}}^{\delta}=\frac{1}{2}<\sigma_{i j}\left(u_{i, j}-\epsilon_{i j}^{T}\right)+\gamma\left(\epsilon^{T}\right)>_{B} .
$$

Here $\gamma\left(\epsilon^{T}\right)$ is the free energy of the phase associated with the transformation strain $\epsilon^{T}$, and the bracket notation indicates volume averages:

$$
<f(x, \delta)>_{B}=\lim _{\delta \rightarrow 0} \frac{1}{|B|} \int_{B} f(x, \delta) d x
$$

For definiteness, our present discussion is restricted to estimation of overall energies at the critical temperature at which the free energies of Austenite and Martensite coincide. We thus take

$$
\gamma\left(\epsilon^{T}\right)=0
$$

for all phases $\epsilon^{T}$; the general case can be handled similarly.

The overall (or homogenized) energy $E$ is defined by the minimum value of $W$ over all admissible stationary distributions of transformation strains. That is

$$
E=\inf _{\epsilon^{T} \in \Lambda} W
$$

where, calling $\epsilon^{T(n)}$ the value of $\epsilon^{T}$ on $G^{(n)}$,

$$
\Lambda=\left\{\epsilon^{T}: \epsilon^{T} \text { is stationary and } \epsilon^{T(n)}(\omega) \in \mathcal{S}^{(n)}(\omega) \text { for all } n \text { and all } \omega\right\},
$$

see (6).

It was shown in [4] that $W$ is given by

$$
\begin{array}{r}
W=\frac{1}{2} \sigma_{i j}^{0} u_{i, j}^{0}-\sigma_{i j}^{0} \epsilon_{i j}^{T(a v)}+\frac{1}{2|B|} \int_{B} C_{i j k l} u_{k, l}^{1}(x, 0) \epsilon_{i j}^{T(a v)} d x \\
-\frac{1}{2|B|} \lim _{\delta \rightarrow 0} \sum_{k, p} \int_{G^{(k), \delta}} \sigma_{i j}^{(p)} \epsilon_{i j}^{T(k)} d x .
\end{array}
$$

Here 0 superindices denote quantities associated with the imposed boundary conditions, $\epsilon_{i j}^{T(k)}$ denotes the transformation strain of the $k$-th grain, $\epsilon_{i j}^{T(a v)}$ denotes the overall average of the transformation strains, and $\sigma_{i j}^{(p)}$ denotes the stress produced by transformation of the $p$-th grain. Except for the stresses $\sigma_{i j}^{(p)}$, all quantities in (7) can be computed explicitly for a given distribution of transformation strains. The stresses $\sigma_{i j}^{(p)}$, on the other hand, can be obtained, from Eshelby's formula, by integration, without need of solving a partial differential equation. It is thus seen that $W$ is a quadratic form in the array of transformation strains $\epsilon^{T}$. The overall energy $E$ associated with given boundary conditions results from $W$ by minimization in the allowable set $\Lambda$ of transformation strains. 


\subsection{Numerics and Bounds}

The expression (7) can be used to derive rigorous upper bounds on the overall energy $E$. Alternatively, statistical optimization methods such as simulated annealing can be used to minimize this expression numerically. A number of interesting conclusions can be drawn by comparison of such bounds and numerical results. We refer to [4] for such considerations and complete details on our approach. In what follows we comment briefly on the nature of our bounds, and we give examples demonstrating the quality of our numerics.

In Figure 3 we present a comparison of numerical results and bounds. In this simplified example the polycrystal is assumed to be a square array of circular grains in two dimensions. (See [4] for details, where a number of additional examples including deviatoric and hydrostatic transformation strains may be found as well.) The "Taylor bound" mentioned in the caption, is the upper bound that results by assumption of a hypothesis of constant strain, such as that one used by G. I. Taylor in the context of polycrystalline plasticity [11]. We note from the figure that, here, the Taylor's hypothesis may lead to errors of the order of $50 \%$. The quantity AE, finally, is the "Austenite Energy", that is, the elastic energy that results in the polycrystal under the same boundary conditions if none of the grains transforms to martensite.
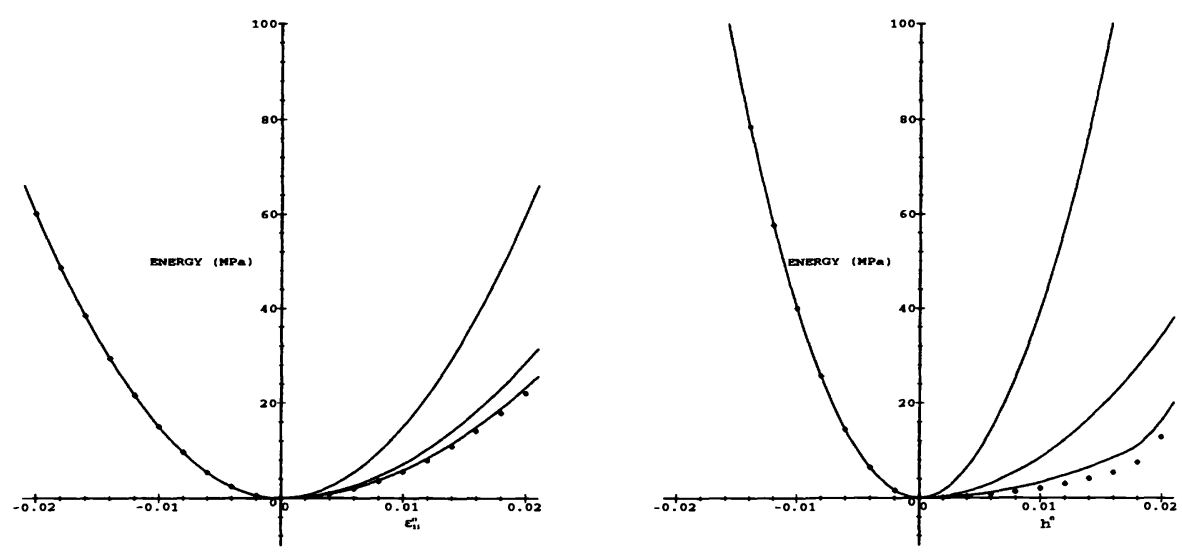

Figure 3: Square array with $2 \%$ hydrostatic transformation strain. Left: uniaxial applied strain $\epsilon_{11}^{0}$; Right: hydrostatic applied strain $h^{0}$. Upper curve: AE; Middle curve: Taylor bound; Lower curve: UG bound; Diamonds: numerical results.

\section{Lenghtscales}

A fundamental element in the development of the crystallographic theory of martensite was the consideration of a planar surface, the habit plane, which separates twinned martensite from untransformed austenite in certain single crystals, cf. [12, p. 170] and [13, p. 1505]. Such configurations certainly do not constitute the only fashion in which austenite and martensite may coexist: a variety of regular as well as irregular patterns generally occur. But habit-plane morphologies are indeed observed in carefully monitored experiments, as demonstrated by a number of compelling micrographs; see [12, p. 82], [1].

Here we follow [13, p. 1505] in their consideration of such especially simple configurations. Our focus is on elasticity and dissipation of elastic energy into other mechanical observables. We present a theory which, based on the existence of the simple patterns mentioned above, attributes the finiteness of the observed microstructures (which would be deemed infinitesimal from an unqualified application of the crystallographic theory) not to competition between elastic and surface energies but, instead, to an interplay between elasticity and energy dissipation. The resulting computations, presented in $\S 3.2$, show quantitative agreement with the observed twin sizes. 


\subsection{Elasticity}

Martensitic transformations give rise to a number of martensite variants, whose associated deformations are characterized by a finite set $\mathcal{T}$ of constant distortions $\mathcal{T}=\left\{\Gamma^{T(1)}, \ldots, \Gamma^{T(n)}\right\}$. The tensors $\Gamma^{T(k)}$ are defined by

$$
\Gamma_{i j}^{T(k)}=\partial_{j} D_{i}^{k}-I_{i j}
$$

where $I$ is the identity tensor and where $D^{i}$ is the homogeneous deformation associated with transformation into the $i$-th variant. Note that, with this convention, $\Gamma^{T(k)}=0$ would indicate null deformation. Also, the tensors $\Gamma^{T}=\Gamma_{i j}^{T}$ need not be symmetric; their symmetric parts equal the corresponding Bain strains $\epsilon^{T}$ :

$$
\epsilon_{i j}^{T}=\frac{1}{2}\left(\Gamma_{i j}^{T}+\Gamma_{j i}^{T}\right)
$$

As pointed out in [13, p.1511], the set $\mathcal{T}$ must be invariant under conjugation by rotations in the symmetry group of the parent phase.

In a general configuration, the transformation distortions vary in space, and the spatial distribution of such quantities within the body is given by a tensor valued function $\gamma^{T}=\gamma^{T}(r), r=(x, y, z)$. The actual transformation distortion $\gamma^{T}(r)$ at a given point in the material is not necessarily given by an element of $\mathcal{T}$, since the phase transition may give rise to relative rotations between the various parts of the body [13]. Further, the true deformation at a point $r$ in the material is generally not equal to $\gamma^{T}(r)$, since additional (small) elastic deformations occur as a result of transformation of the various parts of the body. Thus, the true displacement vector $u=u(r)$ at a point $r$ in the material results as a small elastic deformation that further changes the shape of the material element around point $r$ after it has undergone a distortion of the form

$$
\gamma_{i j}^{T}(r)=R_{i i^{\prime}} \Gamma_{i^{\prime} j}^{T(k)},
$$

for some $k$ and some rotation matrix $R$. The overall deformation vector $D$ is given by $D=D(r)=r+u(r)$.

As we discuss configurations which consist of a planar interface separating austenite on one hand, and fine alternating layers of martensite on the other, we will find it convenient to use coordinates $(x, y, z)$ with the $(x, y)$ coordinate plane orthogonal to both the inter-twin planes and the twin-austenite interface. In such coordinate system the transformation distortions $\gamma^{T}$ are independent of the $z$ variable

$$
\gamma^{T}=\gamma^{T}(x, y)
$$

Assuming, further, that the $x$ axis coincides with the twin-austenite interface, our three dimensional $x$-periodic configuration is described by Figure 4: the plane $y=0$ separates the austenite $y>0$ and the twinned martensite $y<0$ and the $z$ axis is perpendicular to the plane of the figure. The distortion $\gamma^{T}$ vanishes for $y>0$, and it is constant and equal to a rotation of an element of $\mathcal{T}$ in each one of the twin bands in the region $y<0$.

Clearly, the stress $\sigma(r)$ at point $r$ in the material vanishes if $\nabla u(r)=\gamma^{T}(r)$. Thus, for the small elastic deformations that the austenite and the martensite can undergo without additional phase change, it is reasonable to assume a linearly elastic law

$$
\sigma_{i j}(r)=C_{i j k l}(r)\left(\partial_{k} u_{l}(r)-\Gamma_{k l}^{T}(r)\right),
$$

where $C_{i j k l}(r)$ is the stiffness tensor of the phase at point $r$. In view of the relation $C_{i j k l}=C_{i j l k}$ and calling

$$
\epsilon_{i j}=\frac{1}{2}\left(\gamma_{i j}+\gamma_{j i}\right)
$$

we also have

$$
\sigma_{i j}(r)=C_{i j k l}(r)\left(\epsilon_{k l}(r)-\epsilon_{k l}^{T}(r)\right)
$$

Our subsequent analysis assumes isotropic elasticity and identical elastic constants for the austenite and martensite phases, with Lamé constants

$$
\lambda \text { and } \mu \text {, }
$$

where $\mu$ is the shear modulus and where, denoting by $\kappa$ the bulk modulus, $\lambda$ is given by $\kappa=\lambda+\frac{2}{3} \mu$. 


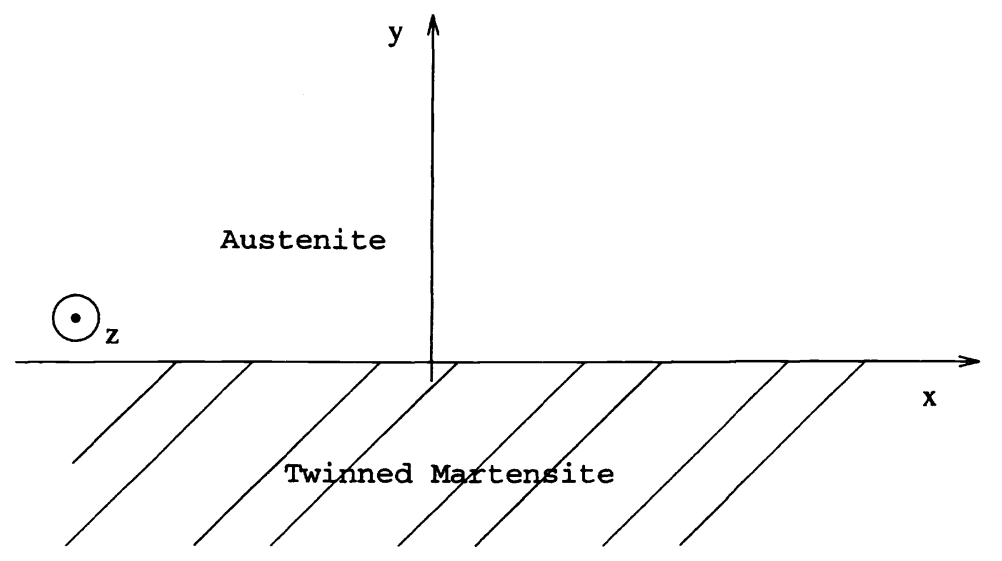

Figure 4: An Austenite-Martensite configuration

Let us now introduce a transformation displacement $u^{T}$ which satisfies

$$
u_{i, j}^{T}=\gamma_{i j}^{T},
$$

and it is continuous outside the interface $y=0$. We take $u^{T}=0$ for $y>0$. To define $u^{T}$ for $y \leq 0$ we note that equations (9) admit a unique solution up to an arbitrary constant. Indeed, on each martensite band we must have

$$
u_{i}^{T}=\gamma_{i j}^{T} x_{j}+\text { const. }
$$

and, in view of the compatibility conditions implied by the crystallographic theory of martensite, it is clear that constants may be chosen to make up a function $u^{T}$ which is continuous in the region $y \leq 0$ and periodic, of period $d$, in the $x$ direction; generically, however, $u^{T}$ is discontinuous at $y=0$. By periodicity, the vector $g(x)=u^{T}\left(x, 0^{-}\right), g=\left(g_{1}, g_{2}, g_{3}\right)$ admits a Fourier series expansion

$$
g(x)=\sum_{\ell=-\infty}^{\infty} g_{\ell} e^{i \alpha_{\ell} x}
$$

with vector coefficients,

$$
g_{\ell}=\left(g_{\ell}^{1}, g_{\ell}^{2}, g_{\ell}^{3}\right)
$$

and with

$$
\alpha_{\ell}=\frac{2 \pi \ell}{d} \text {. }
$$

In an example below we will assume a simplified configuration in which both types of twins have the same width. In this case the Fourier coefficients are given by

$$
g_{\ell}=\left(t_{1}, t_{2}, t_{3}\right) \frac{\left((-1)^{\ell}-1\right)}{\pi \ell \alpha_{\ell}}
$$

for a certain vector $t$. Note that in this case we have

$$
\left|g_{\ell}^{j}\right|^{2}=g_{\ell}^{j} g_{-\ell}^{j}=\left|t^{j}\right|^{2} \frac{d^{2}\left|\left((-1)^{\ell}-1\right)\right|^{2}}{4 \pi^{4} \ell^{4}}
$$

Calling

our problem then becomes

$$
\tilde{v}=u-u^{T}
$$

$$
\partial_{j} C_{i j k l}(r) \tilde{v}_{k, l}(r)=0
$$


with the boundary conditions

$u$ is everywhere continuous and $\left[C_{i j k l}(r) \tilde{v}_{k, l} n_{j}\right]=0$ on $y=0$.

Equivalently,

$$
[\tilde{v}]=g(x) \text { and }\left[C_{i j k l}(r) \tilde{v}_{k, l} n_{j}\right]=0
$$

It can be shown that, by consideration of stresses $\sigma^{p}$ corresponding to a problem of plane strain and a stress $\sigma^{a}$ corresponding to an anti-plane shear problem, the $3 \times 3$ system (8) can be reduced to a decoupled pair of scalar equations in the plane.

For simplicity, we compute the elastic energy in the antiplane shear case only. Assuming transformation strains equal in magnitude to those observed in experiment, we then use our antiplane-shear energy expression in a calculation of the twin widths. Naturally, only order of magnitude predictions should be expected from this analysis. Complete results for the general case will be presented elsewhere.

It is easy to check that, calling $w=\tilde{v}^{3}$, in the antiplane-shear case under consideration we have Fourier expansions

$$
w=\left\{\begin{array}{l}
\sum_{\ell=-\infty}^{\infty} \frac{g_{\ell}^{3}}{2} e^{i \alpha_{\ell} x-\left|\alpha_{\ell}\right| y} \text { for } y>0 \\
-\sum_{\ell=-\infty}^{\infty} \frac{g_{\ell}^{3}}{2} e^{i \alpha_{\ell} x+\left|\alpha_{\ell}\right| y} \text { for } y<0 .
\end{array}\right.
$$

The total elastic energy in the configuration of Figure 4 (per unit length in the $x$ - and $z$-directions) is given by

$$
W=\frac{1}{2 d} \int_{-\infty}^{\infty} d y \int_{0}^{d} \sigma_{i j}\left(\epsilon_{i j}-\epsilon_{i j}^{T}\right) d y
$$

For our specific example this reduces to

$$
\frac{\mu t^{2} d}{4 \pi^{3}} \sum_{\ell=-\infty}^{\infty} \frac{\left((-1)^{\ell}-1\right)^{2}}{|\ell|^{3}}
$$

where $t$ is the projection of the strain of transformation on the habit plane. The infinite sum in this expression equals approximately 8.4 , so that the energy is

$$
0.068 \mu t^{2} d \text {. }
$$

Estimating $t$ by a magnitude of the transformation strain, we find from [5] $t \approx 0.013$. With regards to the elastic modulus, we follow [5] and use $\mu=10^{(11)} \frac{d y n}{c m^{2}}$.

\subsection{Twin widths}

The calculation of the twin widths now proceeds from three main assumptions: 1) Energy dissipation is associated with the formation of the inter-twin interfaces, and this is the only dissipative mechanism in the experiment; 2) The first occurrence of an austenite-martensite interface is as indicated in Figure 5. That is, calling $b$ the side of the square base in the tetragonal bar of Figure 5, a fully formed austenite-martensite interface of length $b \sqrt{2}$, and corresponding twins filling a triangular region $T$, arise not as a propagation of a continuously moving interface, but, instead, as a single nucleation event. After this nucleation event the austenite-martensite interface moves continuously (as observed experimentally). And, 3) The forementioned triangular configuration is formed exactly when the excess energy obtained by cooling in the triangular region reaches a value consistent with the formation of a austenite-martensite interface (which stores a certain amount of energy in elasticity) as well as the formation of the inter-twin interfaces (which, we have postulated, involves dissipation).

We provide some justification for assumptions 1) and 2); assumption 3) appears quite natural but will not otherwise be discussed. With regards to 1 ), clearly no dissipation occurs in the austenite region. If the main energy dissipation took place at the twin-autenite interface, on the other hand, then such dissipation would diminish with diminishing twin widths, and would be very small if the twin widths are small. Of course, this does not rule out the possibility that dissipation takes place both at the austenite-martensite interface, and at the inter-twin interfaces. We think this possibility unlikely 


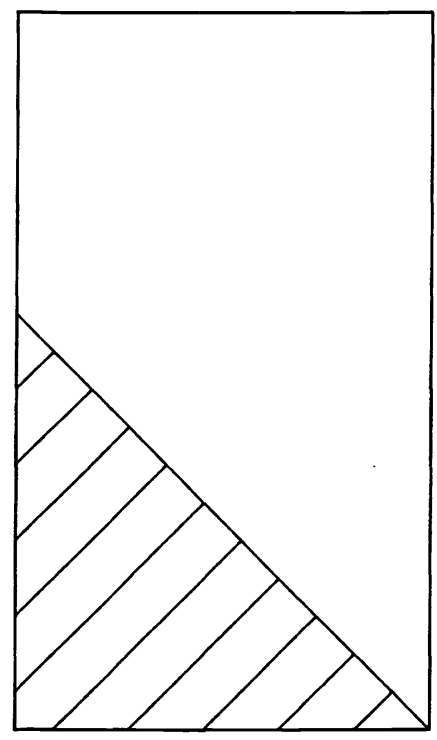

Figure 5: Twins within a bar

in the Indium-Thallium configurations of [5], in view of their small transformation strains. A better understanding in this connection, however, will probably require further experimental investigations.

Assumption 2), on the other hand, is based on the following simple symmetry argument. If a small interface develops on the left hand corner of the bar of Figure 5, then a corresponding interface should develop on the right hand corner as well. As these interfaces grow they would intersect each other, and eventually produce an $X$-interface -which is crystallographically admissible, and experimentally observed in some circumstances $[5,1]$. In the bars considered in these experiments, however, such $X$ interfaces tend to occur in specimens which have been mishandled, or which have not been annealed properly. As reported in $[5,1]$, in well annealed bars of dimensions of those used in these papers, $X$ interfaces tend not to occur. Single nucleation events involving regions substantially larger than the one of Figure 5 would probably not have gone unnoticed, on the other, hand, an therefore our assumption 2) seems fairly well substantiated.

A calculation of the twin widths on the basis of these hypothesis follows easily, now, from the results of the previous section. The energy $W$ of the twin-austenite interface per unit length of interface and per unit length in the $z$ direction equals

$$
W=A d
$$

with $A=0.068 \mu t^{2}$. The total length of twin-twin interfaces in Figure 5, on the other hand, is given by $\frac{s^{2}}{2 d}$, where $s$ is the length of the twin-austenite interface. It follows that the energy dissipated in the formation of this configuration can be expressed in the form

$$
E_{d}=\frac{s^{2}}{2 d} Q
$$

where $Q$ is the total dissipated energy per unit length of twin-twin interface. Thus, using assumption 3), the configuration of Figure 5 may not have been formed unless the triangular region $T$ was cooled in such a way that an amount

$$
E=A d s b+\frac{s^{2}}{2 d} Q
$$

of excess energy was made available within $T$. The configuration will form as soon as the available energy in the triangular region reaches a value equal to the minimum of equation (13) for all positive values of $d$. Differentiation shows that this minimum is achieved when

$$
d=E_{d} \frac{1}{A s b}
$$




$$
d=\sqrt{\frac{s Q}{2 A b}}
$$

It follows that the minimum energy value is

$$
\sqrt{\frac{2 Q A}{b}} s^{\frac{3}{2}}
$$

(Notice, parenthetically, that the energy needed to form a small triangular region scales with $s^{\frac{3}{2}}$, which is much larger than the area $s^{2} / 4$ of the corresponding triangle, (and thus the energy contained in the triangle) for sufficiently small $s$. This remark provides further support for our assumption 2), in that it establishes that some triangular configuration must be nucleated as a single event.)

These are our main results. We now evaluate the expression (14) for parameter values corresponding to the indiumthallium system of [5].

From Figure 8 of $[5, \mathrm{p} .1521]$ the hysteresis width equals $1.6 * 10^{3} \frac{\mathrm{gr}}{\mathrm{cm}^{2}}$, so that the dissipative force equals $1.6 * 10^{3} * b^{2} \frac{\mathrm{gr}}{\mathrm{cm}}$, or equivalently, $1.6 * 10^{6} * b^{2} \frac{d y n}{c m^{2}}$. Half of the hysteresis force times the strain should give a reasonable estimate for the dissipation per unit length of bar on the cooling transformation. Since from [5, p. 1520] the total elongation of the bar is $0.37 \%$, the total dissipation per unit length of bar equals

$$
0.8 * 10^{6} * b^{2} \frac{d y n}{c m^{2}} * 0.37 * 10^{(-2)}
$$

Because the area of the triangular region equals $\frac{b^{2}}{2}$, the dissipation involved in its formation equals one half the dissipation involved in propagating the austenite-twin interface by an amount $b$. So, the dissipation involved in the formation of triangular region equals

$$
E_{d}=0.4 * 10^{6} * b^{3} \frac{d y n}{c m^{2}} * 0.37 * 10^{(-2)}
$$

Since $s=b \sqrt{2}, \mu=10^{(11)}$ (cf. [5]), and using $t=\epsilon=0.013$ and $b=0.5 \mathrm{~cm}$ equation (14) gives

$$
d=\frac{1.48 * 10^{3} \frac{d y n}{c m^{2}} b}{0.068 \sqrt{2} * \mu * t^{2}}
$$

or

$$
d=4.6 \mu m
$$

and the twin width is therefore equal to

$$
\frac{d}{2}=2.3 \mu m
$$

Roughly, this value is in agreement with the experimental observation of [5], which gives a twin width of about $5 \mu m$.

\section{References}

[1] Z. S. Basinski and J. Christian, "Experiments on the martensitic transformation in single crystals of indium-thallium alloys," Acta Met., Vol. 2, pp. 148-166, 1954.

[2] O. P. Bruno, "Quasi-static dynamics and pseudoelasticity in polycrystalline shape-memory wires", Smart Materials and Structures, Vol 4, pp. 7-13, 1995; see also Mathematics and Control in Smart Structures, Proc. SPIE, Vol. 2192, pp. 370-379, 1994.

[3] O. P. Bruno, P. Leo and F. Reitich "Free boundary conditions at austenite-martensite interfaces", Phys. Rev. Lett., Vol. 74, pp. 746-749, 1995.

[4] O. P. Bruno, F. Reitich and P. Leo "The overall elastic energy of polycrystalline martensitic solids", J. Mech. Phys. Solids, Vol. 44, pp. 1051-1101, 1996. 
[5] M. W. Burkart and T. A. Read "Diffusionless phase change in the Indium-Thallium system", Phys. Rev. Lett., Vol. 74, pp. 746-749, 1995.

[6] J. L. Ericksen, "Equilibrium of bars," Journal of Elasticity, Vol. 5, pp. 191-201, 1975.

[7] P. H. Leo, T. W. Shield and O. P. Bruno, "Transient heat transfer effects on the pseudoelastic behavior of shape-memory wires," Acta metall. mater., Vol. 41, pp. 2477-2485 1993.

[8] G. Meyer, SIAM J. Numer. Anal. 10, 522 (1973).

[9] J. A. Shaw and S. Kyriakides, "Thermomechanical aspects of NiTi," J. Mech. Phys. Solids, Vol. 43, pp. 1243-1281, 1995.

[10] T. W. Shield, P. H. Leo and W. C. Grebner, "Quasi-static extension of shape-ememory wires under constant load," Acta Materialia, Vol. 45, pp. 67-74, 1997.

[11] G. I. Taylor, "Plastic strain in metals," J. Inst. Metals, Vol. 62, pp. 307-324, 1938.

[12] C. M. Wayman, "Introduction to the crystallography of martensitic transformations," Macmillan, 1964.

[13] M. S. Wechsler, D. S. Lieberman and T. A. Read, "On the theory of the formation of martensite," Trans. AIME, pp. 1503-1529, 1953. 